\title{
Development of Morphogen Gradients with Spatially Varying Degradation Rates
}

\author{
Hamid Teimouri, Behnaz Bozorgui, and Anatoly B. Kolomeisky* \\ Department of Chemistry and Center for Theoretical Biological Physics, Rice University, \\ Houston, TX 77005-1892 \\ E-mail: tolya@rice.edu
}

\footnotetext{
*To whom correspondence should be addressed
} 


\begin{abstract}
Successful biological development via spatial and temporal regulations of cell differentiation relies on action of multiple signaling molecules that are known as morphogens. It is now well established that biological signaling molecules create non-uniform concentration profiles, called morphogen gradients, that activate different genes, leading to patterning in the developing organisms. The current view of the formation of morphogen gradients is that it is a result of complex reaction-diffusion processes that include production, diffusion and degradation of signaling molecules. Recent studies also suggest that the degradation of morphogens is a critically important step in the whole process. We develop a theoretical model that allows us to investigate the role of a spatially varying degradation in the formation of morphogen gradients. Our analysis show that the spatial inhomogeneities in degradation might strongly influence the dynamics of formation of signaling profiles. Physical-chemical mechanisms of the underlying processes are discussed.
\end{abstract}




\section{Introduction}

Development of various living organisms from initially very small group of identical embryo cells is one of the most fascinating and complex processes in biology. ${ }^{1-4} \mathrm{~A}$ critical stage in biological development is a pattern formation during which the eventual fates of cells become determined at different times and different positions. Several classes of signaling molecules, known as morphogens, play the central role in tissue patterning and organ formation. ${ }^{1-4}$ The term morphogen was first introduced by A. Turing in his seminal paper on mathematical modeling of biological pattern formation. ${ }^{5}$ It is now widely accepted that the concentration gradient of morphogens provide cells with required positional information to activate or inhibit specific genes, probably utilizing the local concentration thresholds and/or other related mechanisms. ${ }^{1-4,6-8}$ In recent years, there has been a substantial progress in experimental and theoretical studies of the mechanisms of embryonic development stimulated by the various morphogen gradients. $6,7,9,24$ However, many features of the biological development processes still remain not well understood. ${ }^{7,8}$

Several mechanisms have been proposed for explaining how the morphogen gradients are established. ${ }^{7-10}$ The simplest and widely popular approach for the description of the signaling profiles formation is called a Synthesis-Diffusion-Degradation (SDD) model. ${ }^{7}$ According to this model, morphogens are synthesized at specific locations, and from the source region they diffuse through a field of embryo cells where they eventually are degraded after binding to cell receptors. ${ }^{7}$ The balance between the synthesis, diffusion and degradation processes leads to the formation of concentration profiles of signaling molecules. Recent investigations also point out to the importance of the degradation steps in the establishment of morphogen gradients. ${ }^{11,12,19,20}$ Without a constant removal of the morphogen molecules, non-uniform concentration profiles cannot be achieved, and thus the proper biological signals cannot be transferred downstream to genetic networks. It was argued that the degradation functions as an effective potential that drive the signaling molecules away from the local source production. ${ }^{11,20}$ So far, most theoretical studies of the formation of morphogen gradients assumed that the degradation rates are constant and uniform, i.e, independent of the spatial positions in the embryo. ${ }^{7,11,12,19,20}$ However, this assumption probably 
is not very realistic since the density of the cell receptors might vary along the field of embryo cells. ${ }^{29}$ Such spatial inhomogeneities might alter the morphogen fluxes absorbed by the cell receptors. But the effect of the spatial variations in degradation on the mechanisms of the formation of signaling profiles is not known.

In this paper, we extend the existing theoretical methods to investigate the role of spatially varying degradation rates on dynamics of morphogen gradients formation. To illustrate our approach, we investigate in detail two examples of the discrete-state SDD model with spatially varying degradation rates for which full analytical solutions can be obtained. Our analysis shows that spatial inhomogeneity in degradation rates have dramatic effects on concentration profiles and on dynamics of their formation. These observations are explained using our original idea of degradation functioning as an effective potential. ${ }^{20}$

\section{Theory}

Let us start the analysis by considering a general discrete-state stochastic SDD model in one dimension with position-dependent degradation rates as shown in Fig. 1. A system consisting of $L+1$ embryo cells distributed sequentially along one-dimensional interval will be investigated. We assume that signaling molecules are produced with a rate $Q$ only at the site $n=0$. From any site $n \geq 0$, they can diffuse in both directions with a diffusion rate $u$. The particles might be degraded at any site $0 \leq n \leq L$ inside the finite interval with a corresponding rate $k_{n}$. Generally, the values of these degradation rates are position-dependent. One can define a function $P_{n}(t)$ as a probability to find the morphogen molecule at the site $n$ at time $t$. The temporal evolution of this probability function is governed by the following master equations:

$$
\frac{d P_{0}(t)}{d t}=Q+u P_{1}(t)-\left(u+k_{0}\right) P_{0}(t)
$$


for $n=0$;

$$
\frac{d P_{n}(t)}{d t}=u\left[P_{n-1}(t)+P_{n+1}(t)\right]-\left(2 u+k_{n}\right) P_{n}(t)
$$

for $n>0$; and

$$
\frac{d P_{L}(t)}{d t}=u P_{L-1}(t)-\left(u+k_{L}\right) P_{L}(t)
$$

for $n=L$. For the case of constant and uniform degradation rates, $k_{n}=k$, this discrete-state SDD model with a linear degradation can be solved exactly. ${ }^{11}$ In a more general scenario, the degradation rates on different spatial positions are not the same. It is difficult to obtain full analytical solutions for arbitrary spatial variations in the degradation rates. However, it is possible to solve the corresponding master equations for several simple cases. In the following sections, we will analyze such two examples. First, we study a discrete-state stochastic SDD model with a local inhomogeneity, i.e., the degradation rate only at one site is different from other sites. In the second case, we consider a more complicated model in which a signaling domain is composed of two regions with different degradation rates. These inhomogeneous degradation models allow us to clarify many aspects of the mechanisms of the establishment of the signaling profiles.

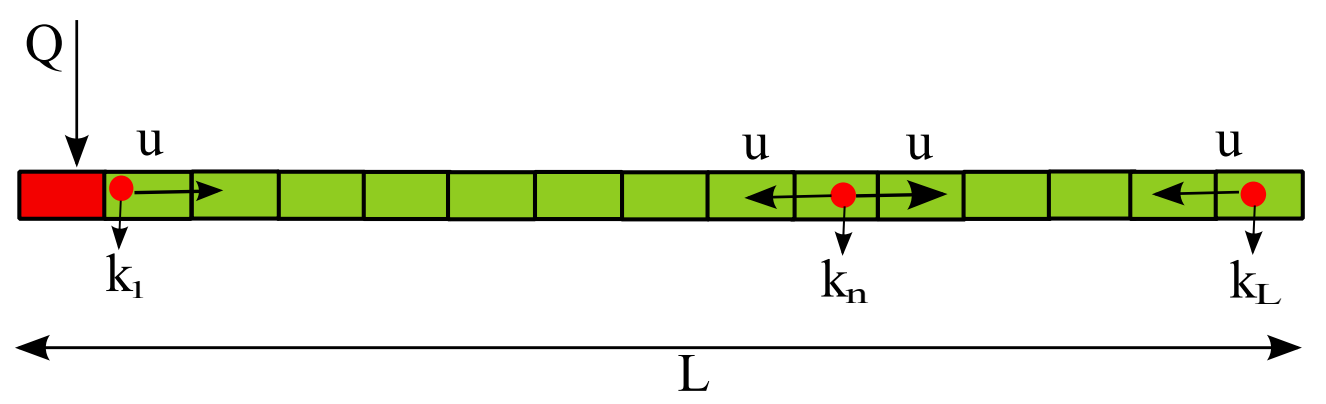

Figure 1: A schematic view of the discrete-state SDD model for the establishment of morphogen gradients on one-dimensional lattice of cells. Degradation rates $k_{n}$ are position-dependent and for each lattice site $n$. Morphogens are produced at the origin, $n=0$, with the rate $Q$. They diffuse along the interval of length $L$ with the rate $u$.

It is important to note that we do not assume that the total number of particles in our system is 
fixed. Because of the open boundary conditions this number can fluctuate. Thus, the probabilities that we compute can only be understood as mean concentrations. ${ }^{30}$

One of the main functions of the morphogen gradients is to transfer biological information to embryo cells. It is still a debate on how it happens at the molecular level, and if the transfer of information is taking place in the stationary state or before reaching the steady-state conditions. ${ }^{8}$ Let us assume for simplicity that the signaling profiles should reach the stationary state for transferring the information. However, our analysis can be also generalized for pre-steady-state coding possibilities. Then the important characteristics of the dynamics of morphogen gradients formation are times needed to achieve the steady-state concentration levels at specific spatial locations. These times are known as local accumulation times (LAT), and a theoretical framework for computing these quantities has been developed recently. ${ }^{12}$ It can be done by utilizing local relaxation functions, which are defined as

$$
R(n, t)=\frac{P(n, t)-P^{(s)}(n)}{P(n, t=0)-P^{(s)}(n)}=1-\frac{P(n, t)}{P^{(s)}(n)} .
$$

The physical meaning of these functions is that they represent the relative distance to the stationary state: at $t=0$ the distance is one, while at steady state it is equal to zero. The explicit formulas for the local accumulation time can be derived then via Laplace transformations of the local relaxation function, $\widetilde{R}(n, s)=\int_{0}^{\infty} R(n, t) e^{-s t} d t,{ }^{12}$

$$
t(n)=-\int_{0}^{\infty} t \frac{\partial R(n, t)}{\partial t} e^{-s t} d t=\widetilde{R}(n, s=0)
$$

This approach can be used to calculate essentially all dynamic properties of the morphogen gradients. 


\section{Results and Discussions}

\section{Finite interval with a local inhomogeneity}

We first consider the simplest situation with a local inhomogeneity in the degradation rates. In this case, the particles might be degraded at any site $n \neq m$ inside the finite interval $0 \leq n \leq L$ with the rate $k$. While the degradation rate at the special site $m$ is different, $k^{\prime} \neq k$. We define again $P_{n}(t)$ as the probability to find the signaling molecule at the site $n$ at time $t$. This probability function is controlled by the following master equations:

$$
\frac{d P_{0}(t)}{d t}=Q+u P_{1}(t)-(u+k) P_{0}(t)
$$

for $n=0$;

$$
\frac{d P_{n}(t)}{d t}=u\left[P_{n-1}(t)+P_{n+1}(t)\right]-(2 u+k) P_{n}(t)
$$

for $n \neq m$;

$$
\frac{d P_{m}(t)}{d t}=u\left[P_{m-1}(t)+P_{m+1}(t)\right]-\left(2 u+k^{\prime}\right) P_{m}(t)
$$

for $n=m$; and

$$
\frac{d P_{L}(t)}{d t}=u P_{L-1}(t)-(u+k) P_{L}(t)
$$

for $n=L$.

At large times, we have $\frac{d P_{n}(t)}{d t}=0$, and these equations can be solved analytically. One can find the solution by using the following guess,

$$
P_{n}^{(s)}= \begin{cases}A_{1} x^{n}+B_{1} x^{m-n}, & \text { for } 0 \leq n \leq m \\ A_{2} x^{n-m}+B_{2} x^{L-n}, & \text { for } m<n \leq L\end{cases}
$$

with $x=\left(2 u+k-\sqrt{k^{2}+4 u k}\right) / 2 u$; and four unknown coefficients $A_{1}, A_{2}, B_{1}$ and $B_{2}$ should be determined from the boundary conditions $(n=0, n=m$ and $n=L)$. Since there are four coefficients, we need four equations to find them. The corresponding master equation for $n=m+1$ yields one 
more necessary equation:

$$
\frac{d P_{m+1}(t)}{d t}=u\left[P_{m}(t)+P_{m+2}(t)\right]-(2 u+k) P_{m+1}(t)
$$

Now, using Eqs. 6, 8, 9, and 11, we can explicitly estimate the coefficients $A_{1}, B_{1}, A_{2}$, and $B_{2}$.

(a) $\mathbf{u}=\mathbf{0 . 0 1}$

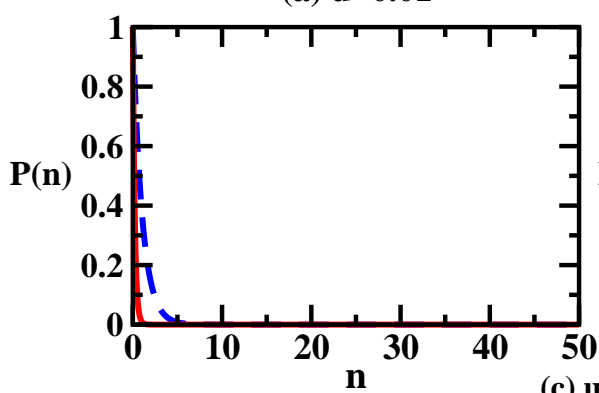

(c) $\mathrm{u}=\mathbf{1 0 0}$

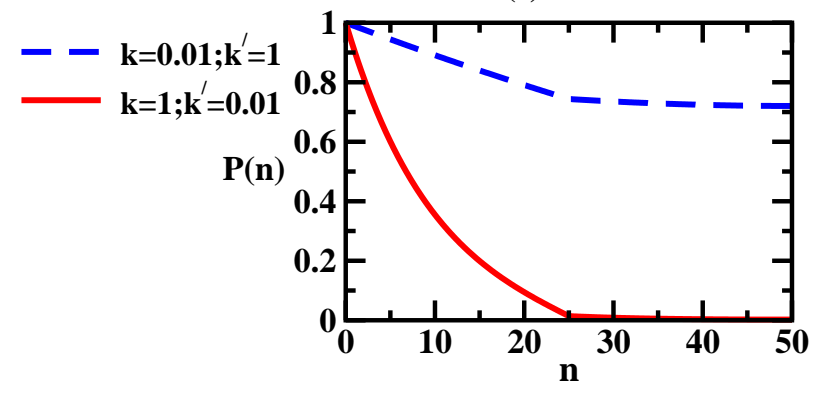

(b) $\mathbf{u}=1$

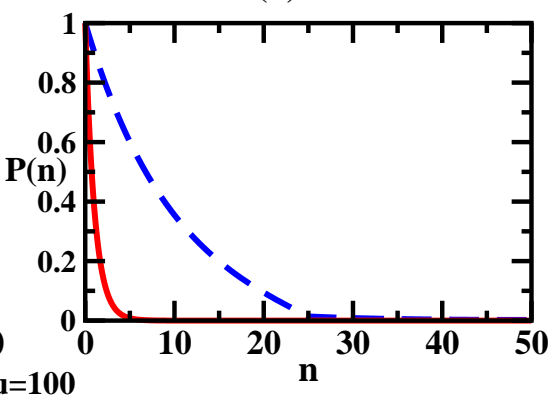

$$
\begin{array}{r}
-1 \\
-1 \\
-1 \\
50
\end{array}
$$

Figure 2: Stationary state density profiles as a function of the distance from the source for the system with a local inhomogeneity in the degradation rates. Solid curves correspond to $k=1 ; k^{\prime}=$ 0.01. Dashed curves correspond to $k=0.01 ; k^{\prime}=1$. (a) $u=0.01$; (b) $u=1$; and (c) $u=100$. For calculations $m=\frac{L}{2}$ was utilized.

The final expressions for the stationary density profiles have the following forms,

$$
\begin{aligned}
P^{(s)}(n) & =\frac{x^{-n}\left[c^{2} x^{2 L}\left(g x^{2 m}-f x^{2 n}\right)+c u x^{2 L}\left(f x^{2 n}-g x^{2 m}+u x^{2 m}-u x^{2 n}\right)\right]}{\left.b^{3} f x^{2 m+2}+b^{2} x^{2 m+2}\left(c g x^{2 m}+f u-u^{2}\right)-b c \eta-c^{2}(c g+u(u-g)) x^{2(L+m)}\right)} \\
- & {\left[\frac{b x^{2 m-n+2}\left(b\left(g x^{2 m}-f x^{2 n}\right)+u\left(-f x^{2 n}+g x^{2 m}-u x^{2 m}+u x^{2 n}\right)\right)}{b^{3} f x^{2 m+2}+b^{2} x^{2 m+2}\left(c g x^{2 m}+f u-u^{2}\right)-b c \eta-c^{2}(c g+u(u-g)) x^{2(L+m)}}\right] }
\end{aligned}
$$


(a) $\mathbf{u}=\mathbf{0 . 0 1}$

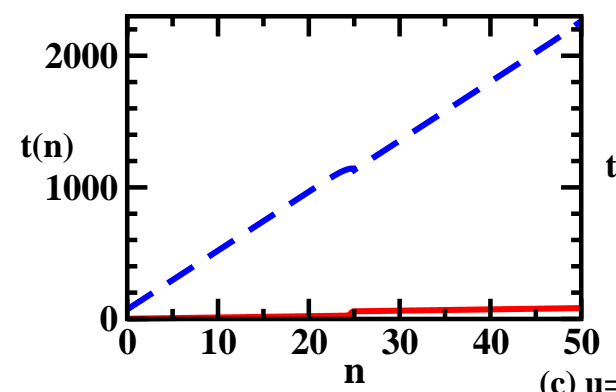

(c) $\mathrm{u}=\mathbf{1 0 0}$

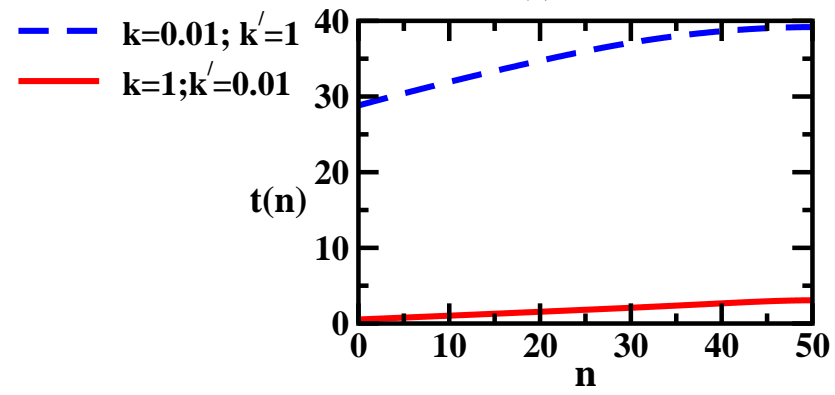

(b) $\mathbf{u}=\mathbf{1}$

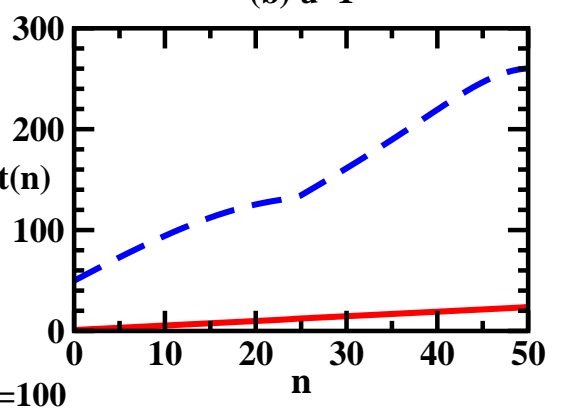

Figure 3: Local accumulation times as a function of the distance from the source for the system with a local inhomogeneity in the degradation rates. Solid curves correspond to $k=1$ and $k^{\prime}=0.01$. Dashed curves correspond to $k=0.01$ and $k^{\prime}=1$. (a) $u=0.01$; (b) $u=1$; and (c) $u=100$. For calculations $m=\frac{L}{2}$ was utilized.

for $0 \leq n \leq m$; and

$$
P^{(s)}(n)=\frac{u(f-g)\left(b x^{2 n}+c x^{2 L}\right) x^{2 m-n+1}}{b^{3} f x^{2 m+2}+b^{2} x^{2 m+2}\left(c g x^{2 m}+f u-u^{2}\right)-b c \eta-c^{2}(c g+u(u-g)) x^{2(L+m)}}
$$

for $m \leq n$. The auxiliary function $\eta$ and the parameters $f, g, b$, and $c$ are given below,

$$
\begin{array}{r}
g=2 u+k^{\prime}-u x^{-1}, \quad f=2 u+k^{\prime}-u x, \\
b=\left(k+\sqrt{k^{2}+4 u k}\right) / 2, \quad c=\left(-k+\sqrt{k^{2}+4 u k}\right) / 2, \\
\eta=-f x^{2 L}-g x^{4 m+2}+u\left(x^{2 L}+x^{4 m+2}\right) .
\end{array}
$$

The resulting morphogen profiles are plotted in Fig. 2 for the inhomogeneity in the middle of the interval, $m=\frac{L}{2}$, and for various diffusion and degradation rates. From the Eqs. (4) and (5) the explicit expressions for the LATs can be calculated. They provide an important information on the 
dynamics of establishing the morphogen gradients. Since the corresponding formulas for LATs are very bulky, we present them in the supplementary material. LAT for the formation of morphogen gradients with $m=\frac{L}{2}$ for various diffusion and degradation rates are presented in Fig. 3. One can see that when the degradation rate at the special site is much smaller than at the other sites $\left(k^{\prime} \ll k\right)$, the effect of the inhomogeneity is local and relatively small. But the strong inhomogeneity $\left(k^{\prime} \gg k\right)$ has a more long-range effect by dividing the interval into two parts with different dynamics and concentration profiles. This agrees well with the idea that degradation behaves like an effective potential. ${ }^{11,20}$ The small degradation rate modifies the system weakly, while the large degradation rate has a more global effect on the system.

\section{Finite interval with two different degradation regions}

A more interesting behavior is observed in the system of two coupled degradation regimes. Here we consider a finite interval of length $L$ composed of two degradation regions of length $m$ and $L-m$. Signaling molecules might be degraded at any site $n$ inside the finite interval $0 \leq n \leq m$ with the rate $k_{1}$. While the degradation rate for any site $m<n \leq L$ is equal to $k_{2}$. Employing again $P_{n}(t)$ as the probability to find the morphogen at site $n$ at $t$, the temporal evolution of the system can be described as,

$$
\frac{d P_{0}(t)}{d t}=Q+u P_{1}(t)-\left(u+k_{1}\right) P_{0}(t)
$$

for $n=0$;

$$
\frac{d P_{n}(t)}{d t}=u\left[P_{n-1}(t)+P_{n+1}(t)\right]-\left(2 u+k_{1}\right) P_{n}(t)
$$

for $0 \leq n \leq m$;

$$
\frac{d P_{n}(t)}{d t}=u\left[P_{n-1}(t)+P_{n+1}(t)\right]-\left(2 u+k_{2}\right) P_{n}(t)
$$

for $m<n<L ;$ and

$$
\frac{d P_{L}(t)}{d t}=u P_{L-1}(t)-\left(u+k_{2}\right) P_{L}(t)
$$


for $n=L$. In the stationary-state regime, we have $\frac{d P_{n}(t)}{d t}=0$, and these equations can be solved analytically. The solution is again can be written as

$$
P_{n}^{(s)}= \begin{cases}A_{1} x_{1}^{n}+B_{1} x_{1}^{m-n}, & \text { for } 0 \leq n \leq m ; \\ A_{2} x_{2}^{n-m}+B_{2} x_{2}^{L-n}, & \text { for } m<n \leq L .\end{cases}
$$

with $x_{1}=\left(2 u+k_{1}-\sqrt{k_{1}^{2}+4 u k_{1}}\right) / 2 u$ and $x_{2}=\left(2 u+k_{2}-\sqrt{k_{2}^{2}+4 u k_{2}}\right) / 2 u$. To determine the unknown coefficients $A_{1}, A_{2}, B_{1}$, and $B_{2}$, we again need four equations. Two of them are given by master equations for $n=0$ and $n=L$. The additional two expressions are master equations for $n=m$ and $n=m+1$,

$$
\begin{gathered}
\frac{d P_{m}(t)}{d t}=u\left[P_{m-1}(t)+P_{m+1}(t)\right]-\left(2 u+k_{1}\right) P_{m}(t) \\
\frac{d P_{m+1}(t)}{d t}=u\left[P_{m}(t)+P_{m+2}(t)\right]-\left(2 u+k_{2}\right) P_{m+1}(t) .
\end{gathered}
$$

After some algebra, we finally obtain the following expressions for the stationary profiles,

$$
\begin{gathered}
P^{(s)}(n \leq m)=\frac{Q\left[B x_{1}^{n}+A x_{1}^{m-n}\right]}{b_{1} B-c_{1} A x_{1}^{m}} ; \\
P^{(s)}(n>m)=\frac{Q\left[\left(B b_{2}\left(u-c_{1}\right) x_{1}^{m}+A\left(u+b_{1}\right) b_{2}\right) x_{2}^{n-m}+\left(B c_{2}\left(u-c_{1}\right)_{1}^{m}+A c_{2}\left(u+b_{1}\right)\right) x_{2}^{2 L-n-m}\right]}{\left[u b_{2} x_{2}+u c_{2} x_{2}^{2 L-2 m-1}\right]\left[b_{1} B-c_{1} A x_{1}^{m}\right]},
\end{gathered}
$$

where the auxiliary functions $A$ and $B$ are specified as

$$
\begin{array}{r}
A=x_{1}^{m}\left[x_{2} b_{2}^{2}\left(u-c_{1}\right)\left(u+b_{2}\right)-u^{2} b_{2}^{2} x_{2}\right]+x_{1}^{m} x_{2}^{2 L-2 m-1}\left[b_{2}\left(u-c_{2}\right)\left(u-c_{1}\right) c_{2}-u^{2} b_{2} c_{2}\right], \\
B=x_{2} b_{2}^{2} u^{2}-x_{2} b_{2}^{2}\left(b_{1}+u\right)\left(b_{2}+u\right)+x_{2}^{2 L-2 m-1}\left[u^{2} b_{2} c_{2}-b_{2}\left(b_{1}+u\right)\left(u-c_{2}\right) c_{2}\right],
\end{array}
$$


(a) $\mathrm{u}=0.01$

(b) $u=1$
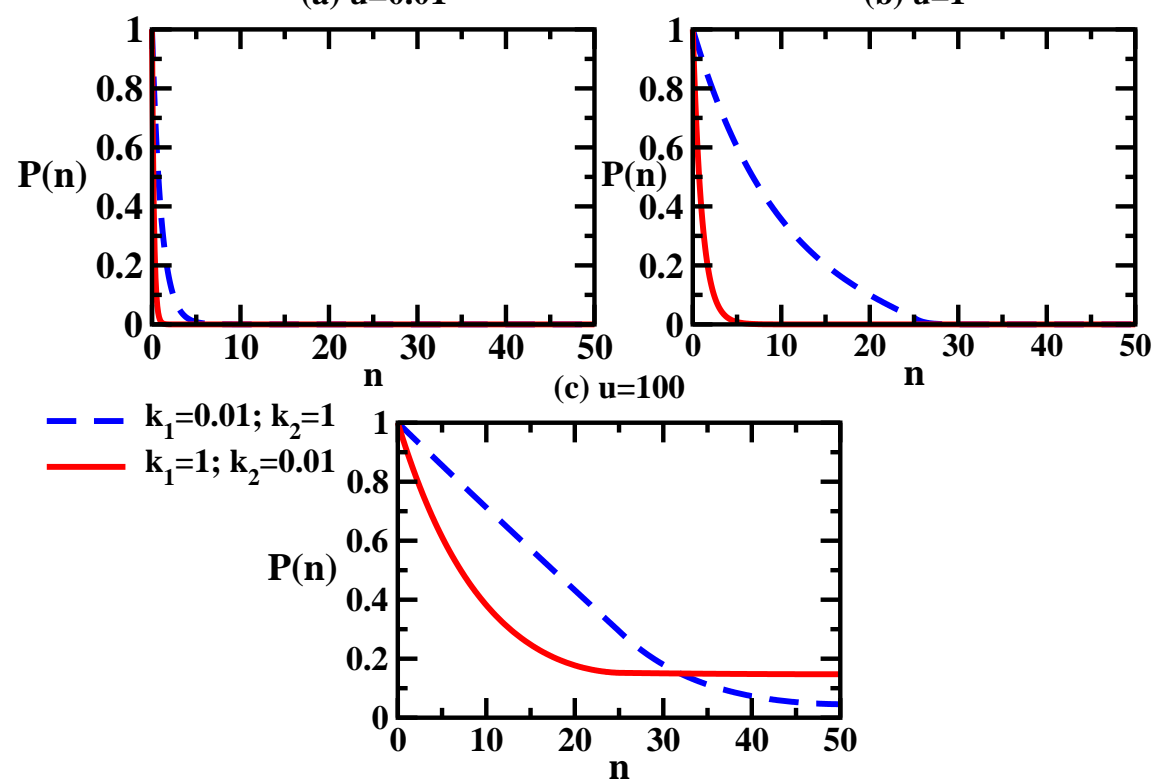

Figure 4: Stationary state density profile as a function of the distance from the origin for the system with two degradation regions. Solid curves correspond to $k_{1}=1$ and $k_{2}=0.01$. Dashed curves correspond to $k_{1}=0.01$ and $k_{2}=1$. (a) $u=0.01$; (b) $u=1$; and (c) $u=100$. For calculations $m=\frac{L}{2}$ was utilized.

with constants $b_{1}, b_{2}, c_{1}, c_{2}, d_{1}, d_{2}, e_{1}$, and $e_{2}$ given by

$$
\begin{gathered}
b_{1}=\left(k_{1}+\sqrt{k_{1}^{2}+4 u k_{1}}\right) / 2, \quad b_{2}=\left(k_{2}+\sqrt{k_{2}^{2}+4 u k_{2}}\right) / 2, \\
c_{1}=\left(-k_{1}+\sqrt{k_{1}^{2}+4 u k_{1}}\right) / 2, \quad c_{2}=\left(-k_{2}+\sqrt{k_{2}^{2}+4 u k_{2}}\right) / 2, \\
d_{1}=\frac{2 u+k_{1}+\sqrt{k_{1}^{2}+4 u k_{1}}}{2 \sqrt{k_{1}^{2}+4 u k_{1}}}, \quad d_{2}=\frac{2 u+k_{2}+\sqrt{k_{2}^{2}+4 u k_{2}}}{2 \sqrt{k_{2}^{2}+4 u k_{2}}}, \\
e_{1}=\frac{2 u+k_{1}-\sqrt{k_{1}^{2}+4 u k_{1}}}{2 \sqrt{k_{1}^{2}+4 u k_{1}}}, \quad e_{2}=\frac{2 u+k_{2}-\sqrt{k_{2}^{2}+4 u k_{2}}}{2 \sqrt{k_{2}^{2}+4 u k_{2}}} .
\end{gathered}
$$

The results of our calculations for density profiles for the system with two degradation regions are presented in Fig. 4. One can see that for slow diffusion (Fig. 4a) there is not much difference in the behavior independently of the order of degradation regions. The degradation is so strong that most morphogens are removed close to the origin. However, the difference becomes more 
(a) $\mathbf{u}=\mathbf{0 . 0 1}$

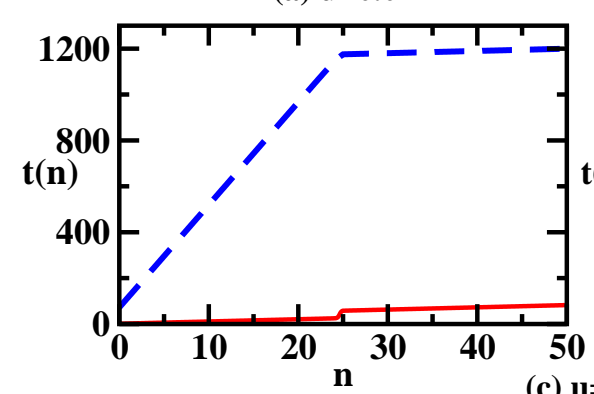

(c) $\mathrm{u}=\mathbf{1 0 0}$
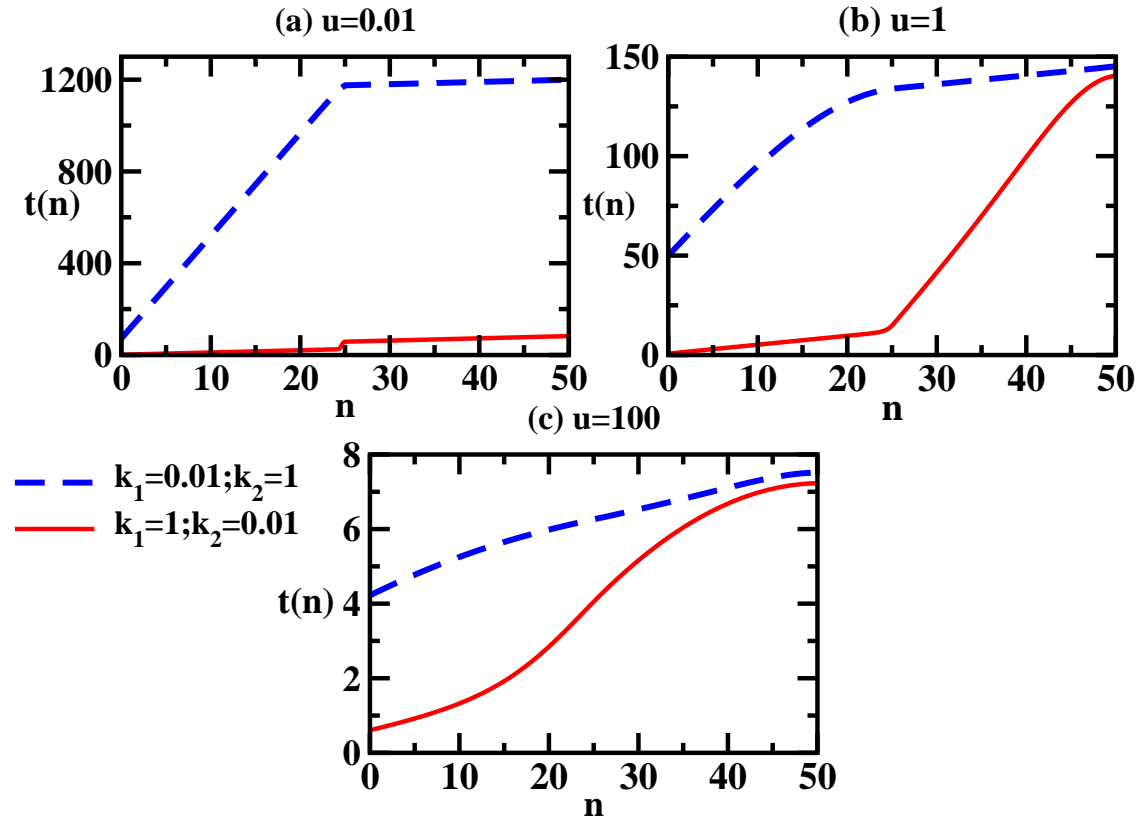

Figure 5: Local accumulation time as a function of the distance from the origin for the system with two degradation regions. Solid curves correspond to $k_{1}=1$ and $k_{2}=0.01$. Dashed curves correspond to $k_{1}=0.01$ and $k_{2}=1$. (a) $u=0.01$; (b) $u=1$; and (c) $u=100$. For calculations $m=\frac{L}{2}$ was utilized.

pronounced with increasing the diffusion rate $u$. For the system with a strong degradation in the first region $\left(k_{1} \gg k_{2}\right)$ there is strong decay in the concentration profile, which is followed by essentially a constant density profile: see Fig 4c. This observation is easy to explain: there is a strong tendency to remove morphogens in the first region, and in the second one the dynamics is closer to a free diffusion. But for the system with the strong degradation in the second region $\left(k_{1} \ll k_{2}\right)$ there is a relatively weak linear decay in the region $0 \leq n \leq m$, and the fast exponential decay in the second region, $m \leq n \leq L$. The second region with strong decay serves as a sink for signaling molecules in the first part, yielding the expected in this case linear profile.

More information about the dynamics of formation of morphogen gradients with spatially varying degradation rates can be obtained from calculations of LAT. The explicit expressions are very bulky, and they are given in the supporting material. The results of LAT for systems with two degradation regions are presented in Fig. 5. Different behavior at two regions is clearly observed. But one can also see that the system with the strong degradation in the first region (red curves in 
(a) $\mathbf{u}=\mathbf{0 . 0 1}$
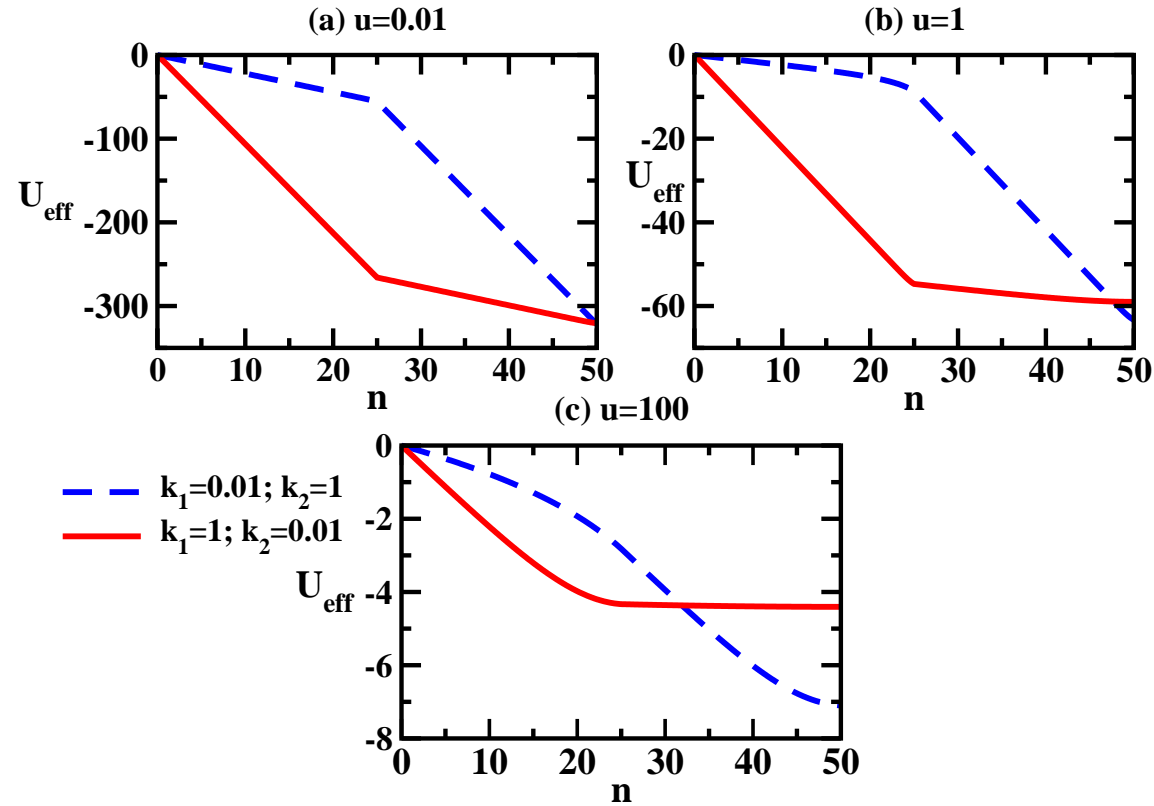

Figure 6: Effective potential due to degradation as a function of the distance from the origin in units of $k_{B} T$ for the system with two degradation regions. Solid curves correspond to $k=1_{1}$ and $k_{2}=0.01$. Dashed curves correspond to $k_{1}=0.01$ and $k_{2}=1$. (a) $u=0.01$; (b) $u=1$; and (c) $u=100$. For calculations $m=\frac{L}{2}$ was utilized.

Fig. 5) always reaches the stationary profiles faster. The strongest effect is observed for slow diffusion (Fig. 5a), while for faster diffusion rates the difference is lower: see Figs. 5b and 5c. This is a surprising result. In our calculations we took $m=L / 2$, i.e., two regions have the same length. The overall amount of degradation is the same in both systems, the difference is only in the order degradation regions. One would naively expect that relaxation dynamics to the stationary state at the end of the interval $(n=L)$ should be the same in both systems. But this is not the case at all regimes. This leads us to an important conclusion that the dynamics of formation of morphogen gradients can be modified by changing the spatial distribution of degradation rates, even without changing the amplitudes of the degradation rates.

To explain these surprising observations we can invoke the idea of effective potential due to degradation that was proposed earlier by us. ${ }^{11,20}$ Let us consider 2 neighboring lattice cites, $n$ and $n+1$. Because the morphogens are produced at the origin before diffusing along the interval, signaling molecules at the site $n+1$ spend more time in the system than particles on the site $n$. Then 
they have higher probability to be removed from the system, the concentration of morphogens is generally smaller at the site $n+1$, creating a gradient. Such concentration gradient can be also obtained as a result of the action of the potential in the system, acting in the direction of increasing $n$, but without degradations. ${ }^{11,20}$ This effective potential can be estimated in terms of the stationary concentrations,

$$
U_{e f f} \simeq k_{B} T \ln P_{n}
$$

We plot such effective potentials in Fig. 6. One can see that the effective potential is always stronger for the system with the strong degradation in the first region (red curves in Fig. 6) for weak diffusion. Increasing the diffusion rate $u$ creates the region where the potential from the system with weaker degradations in the first region becomes stronger (blue curves in Fig. 6). But, on average over the whole interval, the effective potential is stronger for the system with $k_{1} \gg k_{2}$. This nicely explains the dynamics of the relaxation to the stationary states as presented in Fig. 5. LAT is smaller when the effective potentials, that drive morphogens along the interval, are stronger.

\section{Conclusions}

We developed a theoretical framework for investigating the role of spatially varying degradation rates in the formation of morphogen gradients. Our analysis is based on the discrete-state stochastic models of the formation of signaling profiles. The approach provides a full analytical description for the stationary profiles and local accumulation times (LATs). We specifically analyzed two cases of systems with spatially varying degradations. First, a local inhomogeneity model, in which the degradation rate on a single cell differs from all other degradation rates, was considered. Second, we studied a system with two different regions of degradation. Our analysis shows that in both cases the inhomogeneity might lead to strong changes in dynamic behavior of the system. The effect is stronger for slow diffusion, while for the fast diffusion it becomes smaller. We also found a surprising result that the dynamics of morphogen gradient formation can be strongly influenced by varying the spatial distribution of degradation rates without changing the total amount of 
degradation. Using the idea of effective potential created by degradation, all obtained results are fully explained. Our theoretical method suggests that dynamics of signaling processes can be well tuned by modifying not only the strength of the degradation but also a spatial distribution of the receptors.

Although our approach provides a clear physical picture of the underlying processes during the development of morphogen gradients, it should be emphasized that the model is oversimplified and many important features are neglected, including temporal evolution of degradation rates, coupling of reaction-diffusion processes in signaling molecules with underlying mechanical changes in the embryo cells, and many others. It will be important to test our predictions in more advanced theoretical studies as well as directly in experiments.

\section{Supporting Information Available}

The following files are available free of charge.

- paper.ImhomogDegradation-sm.pdf: explicit calculations of local accumulation times This material is available free of charge via the Internet at http://pubs . acs . org/.

\section{Acknowledgement}

The work was supported by the Welch Foundation (Grant C-1559), from the NSF (Grant CHE1360979), and by the Center for Theoretical Biological Physics sponsored by the NSF (Grant PHY-1427654).

\section{References}

(1) Martinez-Arias, A.; Stewart, A.; Molecular Principles of Animal Development; Oxford University Press: New York, 2002.

(2) Lodish, H.; Berk, A.; Kaiser, C.A.; Krieger, M.; Scott, M.P.; Bretscher, A.; Ploegh, H.; Matsudaira, P.; Molecular Cell Biology, 6th ed.; W.H. Freeman: New York, 2007. 
(3) Wolpert, L.; Principles of Development; Oxford University Press: New York, 1998.

(4) Harrison, L.G.; Kinetic Theory of Living Pattern. Cambridge University Press: New York, 1993.

(5) Turing, A.M. The Chemical Basis of Morphogenesis. Philos. Trans. R. Soc. 1952, 237, 37-72.

(6) Kerszberg, M.; Wolpert, L. Specifying Positional Information in the Embryo: Looking Beyond Morphogens. Cell 2007, 130, 205.

(7) Porcher, A.; Dostatni, N. The Bicoid Morphogen System. Curr. Biol. 2010, 20, R249-R254.

(8) Briscoe J.; Small, S. Morphogen Rules: Design Principles of Gradient-Mediated Embryo Patterning. Development 2015, 142, 3996-4009.

(9) Kornberg, T. B. The Imperatives of Context and Contour for Morphogen Dispersion. Biophys. J. 2012, 103, 2252-2256.

(10) Müller, P.; Rogers, K. W.; Yu, S. R.; Brand M.; Schier, A. F. Morphogen Transport. Development 2013, 140, 1621-1638.

(11) Kolomeisky, A. B. Formation of a Morphogen Gradient: Acceleration by Degradation. $J$. Phys. Chem. Lett. 2011, 2, 1502-1505.

(12) Berezhkovskii, A. M.; Sample, C.; Shvartsman, S. Y. How Long Does It Take to Establish a Morphogen Gradient? Biophys. J. 2010, 99, L59-L61.

(13) Eldar, A. ; Rosin, D.; Shilo B-Z.; Barkai, N. Self-enhanced Ligand Degradation Underlies Robustness of Morphogen Gradients. Dev. Cell. 2003, 5, 653-646.

(14) Lander, D. A. Morpheus Unbound: Reimagining the Morphogen Gradient. Cell. 2007, 128, 245-256.

(15) Grimm, O.; Coppy, M.; Wieschaus, E. Modelling the Bicoid Gradient. Development 2010, 137, 2253-2264. 
(16) Gordon, P. V.; Sample, C.; Berezhkovskii, A. M.; Muratov, C. B.; Shvartsman, S. Y. Local Kinetics of Morphogen Gradients. Proc. Natl. Acad. Sci. USA 2011, 108, 6157-6162.

(17) Kicheva, A.; Pantazis, P.; Bollenbach, T.; Kalaidzidis, Y.; Bittig, T.; Jülicher, F.; GonzalesGaitan, M. Kinetics of Morphogen Gradient Formation. Science 2007, 315, 521-525.

(18) Drocco, J.A.; Grimm, O.; Tank, D.W.; Wieschaus, E. Measurement and Perturbation of Morphogen Lifetime: Effects on Gradient Shape. Biophys. J. 2011, 101, 1807-1815.

(19) Teimouri, T.; Kolomeisky, A. B. Development of Morphogen Gradient: The Role of Dimension and Discreteness. J. Chem. Phys. 2014, 140, 085102-14.

(20) Bozorgui, B.; Teimouri, T.; Kolomeisky, A. B. Theoretical Analysis of Degradation Mechanisms in the Formation of Morphogen Gradients. J. Chem. Phys. 2015, 143, 025102-7.

(21) Fedotov, S.; Falconer, S. Nonlinear Degradation-Enhanced Transport of Morphogens Performing Subdiffusion. Phys. Rev. E 2014, 89, 012107-6.

(22) England, J.L.; Cardy, J. Morphogen Gradient from a Noisy Source. Phys. Rev. Lett. 2005, 94, 078101-4.

(23) Drocco, J.A.; Wieschaus, E.; Tank, D.W. The Synthesis-Diffusion-Degradation Model Explains Bicoid Gradient Formation in Unfertilized Eggs. Phys. Biol. 2012, 9, 055004.

(24) Gregor, T.; Wieschaus, E.F.; McGregor, A.P.; Bialek W.; Tank, D.W. Stability and Nuclear Dynamics of the Bicoid Morphogen Gradient. Cell 2007, 130, 141-152.

(25) Berezhkovskii, A. M.; Shvartsman, S. Y. Kinetics of Receptor Occupancy During Morphogen Gradient Formation. J. Chem. Phys. 2013, 138, 244105-7.

(26) Little, S.C.; Tkacik, G.; Kneeland, T.B.; Wieschaus, E.; Gregor, T. The Formation of the Bicoid Morphogen Gradient Requires Protein Movement from Anteriorly Localized mRNA. PLoS Biol. 2011, 9, e1000596. 
(27) Spirov, A.; Fahmy, K.; Schneider, M.; Frei, E.; Noll, M.; Baumgartner, S. Formation of the Bicoid Morphogen Gradient: an mRNA Gradient Dictates the Protein Gradient. Development 2009, 136, 605-614.

(28) Chen, Y.; Struhl, G. Dual Roles for Patched in Sequestering and Transducing Hedgehog. Cell 1996, 87, 553-563.

(29) Reingruber, J.; Holcman, D. Computational and mathematical methods for morphogenetic gradient analysis, boundary formation and axonal targeting. Seminars in Cell and Developmental Biology 2014 35, 189-202.

(30) Heuett, W.J.; Qian, H. Grand canonical Markov model: A stochastic theory for open nonequilibrium biochemical networks. J. Chem. Phys. 2006, 124, 044110. 


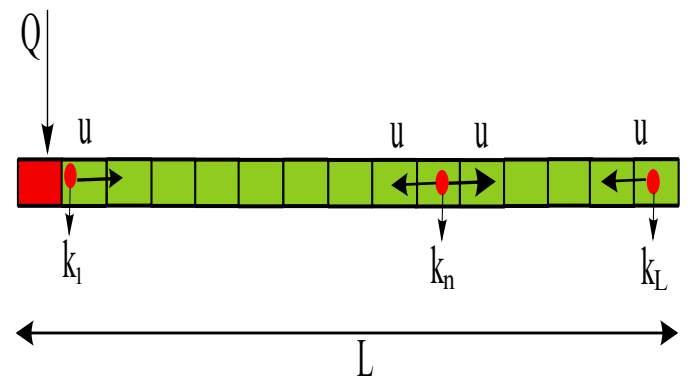

